\title{
Die Senologie aus Sicht der Gynäkologischen Radiologie
}

\section{R. Schulz-Wendtland, Kongresspräsident der 31. Jahrestagung der Deutschen Gesellschaft für Senologie}

Als Salomon im Jahre 1913 aus der chirurgischen Universitätsklinik Berlin zum ersten Mal über die Anwendung der Röntgenstrahlung zur Untersuchung der weiblichen Brusterkrankungen im Archiv für klinische Chirurgie berichtete, galt dies nicht der Einführung einer neuen klinischen Diagnostikmethode, sondern der wissenschaftlichen Erforschung der Karzinomformen und der Karzinomausbreitung. Er hatte Röntgenaufnahmen von Operationspräparaten nach Mammaablatio gemacht und die röntgenologischen Zeichen des Karzinoms studiert. Liest man die erste Publikation über die Mammografie, so ist man erstaunt, wie viel Salomon damals bereits wusste, viel später vergessen wurde und wie wenig hinzuzufügen notwendig war, um den heutigen Stand der Mammografie zu erreichen. Salomon fasst zusammen: „Die Röntgenfotografien exzidierter Mammapräparate geben demonstrable Übersichtsbilder über Ausbreitung und Folgen der Krebse, durch diese Bilder im Verein mit histologischer Untersuchung der verdächtigen Randpartien ergibt sich die Notwendigkeit, mindestens drei Fingerbreit entfernt von der peripher fühlbaren Grenze des Tumors die Excision desselben vorzunehmen“.

Die Gynäkologische Radiologie und der Begriff der Senologie waren im Geist geboren.

Seit Gründung der Deutschen Gesellschaft für Senologie im Jahre 1980 ist die Gynäkologische Radiologie ein fester Bestandteil unserer interdisziplinären Gesellschaft. Es sind insbesondere die Professoren Frischbier, Frischkorn, Hoeffken und von Fournier zu nennen, welche durch Ihre umfassende Ausbildung als Diagnostiker, Operateure und Strahlentherapeuten die Gynäkologische Radiologie zu dieser Zeit prägten.

Durch die Spezialisierung, aber auch durch die immer aufwändigeren und zeitintensiveren Methoden ist der Schwerpunkt der Gynäkologischen Radiologie heute auf die Diagnostik von Brusterkrankungen fokussiert. Die Deutsche Gesellschaft für Senologie hat den Begriff der „Komplementären Mammadiagnostik“ erstmals definiert und in den folgenden drei Jahrzehnten weiterentwickelt. Es wurden in der Mammadiagnostik unter strengen wissenschaftlichen Kriterien neue Methoden eingeführt, revidiert, verlassen oder aber auch nach Jahren wieder neu entdeckt. Die
Gynäkologische Radiologie beinhaltet heute das komplette Spektrum der bildgebenden Mammadiagnostik, bestehend aus digitaler Mammografie mit der jetzigen Möglichkeit der 3D-Bildgebung und Tomosynthese, der 3D- /4D-Ultrasonografie mit dem Zusatz der Elastografie, der 3D 1,5-3-Tesla-MRT-Diagnostik sowie aus den mit diesen Methoden vorgegebenen Möglichkeiten der Intervention, um im Einklang mit dem onkochirurgischen Management einen maximalen Beitrag zur Diagnostik und Behandlung der Patientinnen zu leisten.

Ganz besonders verpflichtet im senologischen Gedanken ist die Gynäkologische Radiologie der Einführung und flächendeckenden Versorgung der Bevölkerung durch ein von allen Kassen finanziertes Mammografiescreening, eine Reihenuntersuchung aller Frauen im Alter von 50 bis 69 Jahren - Frau Dr. Reichel als Pionierin sei besonders erwähnt.

Zukunftsvisionen für die gynäkologische Radiologie sind eine Bildfusion unterschiedlicher Modalitäten einschließlich molekularbiologischer Untersuchungsmethoden und eine maximale fachübergreifende Vernetzung aller Kolleginnen und Kollegen, Institutionen und Einrichtungen, der an der Diagnostik und Therapie onkologischer Patientinnen Beteiligten. Denn auf diesem Weg kann ein optimales Behandlungsergebnis für die Patientin auf höchster Qualitätsstufe erreicht werden - die Brustzentren und Leitlinien sind der erste Schritt dazu.

\section{Bibliografie}

Dol http://dx.doi.org/10.1055/s-0031-1271493

Senologie 2011; 8: 72

(c) Georg Thieme Verlag KG Stuttgart · New York · ISSN 1611-6453

Korrespondenzadresse

Prof. Dr. med. Rüdiger Schulz-Wendtland

Radiologisches Institut

Gynäkologische Radiologie

Universität Erlangen-Nürnberg

Universitätsstr. 21-23

91054 Erlangen

ruediger.schulz-wendtland@uk-erlangen.de 